\section{Séptima Conferencia de "Primeras Damas" de las Américas ${ }^{1}$}

1 Adaptado del informe "Séptima cumbre de las Primeras Damas", presentado a la OPS por María Teresa Cerqueira, Asesora Regional de la OPS en Educación para la Salud y la Participación Social, División de Promoción y Protección de la Salud; y de los documentos sobre las iniciativas "Escuelas siglo XXI: formando para la vida" y "Salud y nutrición escolar en América Latina y el Caribe: desafíos en el nuevo milenio".
Los días 8 y 9 de octubre de 1997 tuvo lugar en la Ciudad de Panamá la Séptima Conferencia de Esposas de Jefes de Estado y de Gobierno de las Américas, en la que participaron las "primeras damas" y otras representantes de más de 30 países, así como de organismos nacionales e internacionales. Estas reuniones se iniciaron en 1990 con objeto de acordar un programa de trabajo que permitiera a las primeras damas apoyar las políticas sociales impulsadas por los gobiernos de sus países respectivos. Las esposas de jefes de Estado y de gobierno han reconocido que muchos países enfrentan problemas comunes, principalmente en relación con la salud y la educación de las mujeres, los niños y los adolescentes, y que por medio del trabajo conjunto de compartir y promover proyectos y programas nacionales y regionales pueden fomentarse el desarrollo y bienestar de los países.

En las conferencias anteriores se iniciaron o se apoyaron los trabajos nacionales en áreas relacionadas con la salud y la educación de dichos grupos de población, y con los acuerdos y compromisos adquiridos por sus países en conferencias y cumbres internacionales. El papel que desempeñan las primeras damas como abogadas de los sectores vulnerables de la sociedad es importante por la influencia que ellas son capaces de ejercer en el público. Están, por lo tanto, en una situación privilegiada para promover un mejor desarrollo humano equitativo tanto en sus países como en el medio regional. A lo largo del decenio de 1990 las recomendaciones emanadas de los foros de primeras damas han motivado a los Gobiernos de las Américas a elaborar planes y programas para alcanzar objetivos económicos y sociales compatibles con la búsqueda de un bienestar social duradero.

Al inaugurar el encuentro, Ernesto Pérez de Balladares, Presidente de Panamá, felicitó a las primeras damas por poner el conjunto de sus talentos e influencia al servicio de las causas sociales. En los comentarios siguientes, el Presidente reconoció que, a pesar de que las mujeres tienen grandes responsabilidades, suelen también tener una voz muy débil en las decisiones políticas. Se pronunció a favor de la plena participación de las mujeres en los ámbitos decisorios de política, economía, educación y otros campos y apoyó un plan de acción basado en la justicia para lograr la igualdad entre hombres y mujeres. La señora de Pérez de Balladares dio una bienvenida especial a las primeras damas visitantes y llamó la atención al hecho de que su interés en los 
problemas del desarrollo humano había hallado eco en la sociedad moderna de América Latina, pues dentro de las prioridades y políticas de cada país se había logrado apoyar planes estratégicos para mejorar la calidad de vida y el bienestar de las poblaciones.

La reina Fabiola de Bélgica expresó su indignación por la miseria en que viven las mujeres rurales de muchos países y recordó los compromisos contraídos por los firmantes en la Declaración de Ginebra. Señaló la triple función que desempeñan la mayoría de las mujeres como madres, aportadoras al sustento de sus familias y voluntarias en actividades comunitarias. Recordando un proverbio africano, declaró que "cuando se educa a un niño se educa a una persona pero, cuando se educa a una niña, se educa a una familia entera y a una nación". Por esa razón, la educación de las niñas es la inversión de mayor rendimiento que puede hacer un país para progresar en su desarrollo.

Por su parte Rebeca Grynspan, vicepresidenta de Costa Rica y conferenciante especial, anunció que su objetivo oculto era convertir a todos los presentes en luchadores por la sostenibilidad democrática. Citando un proverbio indígena, dijo: "la mitad del cielo descansa en nosotras y, si lo soltáramos, se caería". Trayendo a colación las diversas circunstancias de la vida de las mujeres rurales, reclamó contra la falta de políticas públicas que comprendan que su pobreza resulta de su situación laboral, porque son personas invisibles para el sistema. "La mayor injusticia", añadió, "es que cuando tienen éxito los mercados, las gestiones de venta y la comercialización de los productos, las mujeres son desplazadas y los varones sacan provecho de esos éxitos y ganancias". Si bien más de $40 \%$ de la fuerza laboral rural está constituida por mujeres y muchas de ellas sostienen solas sus hogares, su opinión rara vez se tiene en cuenta y la mujer tiene poco acceso a crédito, asistencia técnica o capacitación. Para empezar a lograr algo de igualdad de oportunidad para ambos sexos, será preciso cerrar la brecha que existe entre la legislación y la realidad.

\section{PRESENTACIONES}

Durante las sesiones plenarias se presentaron varios proyectos de programas y se pronunciaron conferencias especiales. El Instituto Interamericano de Cooperación para la Agricultura presentó un programa de apoyo al desarrollo de la mujer rural y la UNESCO propuso otro con el fin de respaldar la educación para los derechos humanos y la cultura de paz. La enviada de las Naciones Unidas pronunció una conferencia sobre el abuso infantil y el Banco Interamericano dedicó un fondo a apoyar el liderazgo de la mujer y prevenir la violencia fami- liar. Además, se presentó una importante iniciativa conjunta del Banco Mundial, el Fondo de las Naciones Unidas para la Infancia, el Fondo de Población de las Naciones Unidas y la OPS para mejorar la salud y la nutrición de los escolares de las Américas. La iniciativa, denominada "Salud y nutrición escolar en América Latina y el Caribe: desafíos en el nuevo milenio", representa un enlace entre la Iniciativa de Escuelas Promotoras de Salud de la OPS/OMS y el Programa de Salud y Nutrición Escolar para Latinoamérica y el Caribe, del Banco Mundial. Se manifestó vivo interés en ese tema y hubo comentarios sobre las actividades similares ya en marcha en algunos países, mientras que otros indicaron su deseo de iniciarlas.

\section{Salud y nutrición escolar en América Latina y el Caribe: desafíos en el nuevo milenio}

Para dar una idea de la iniciativa, vale explicar que se basa en la necesidad urgente de invertir en la salud de los escolares en una época en que América Latina y el Caribe tienen más niños de edad escolar que nunca (más de 220 millones entre los 5 y 18 años de edad). Se fundamenta en el principio de que la buena salud es esencial para poder aprender, promueve el desarrollo intelectual, emocional y físico y contribuye a la asistencia regular. Además, los programas basados en las escuelas aprovechan la estructura ya existente para llegar a un grupo de población fácilmente accesible, con la ayuda de una fuerza laboral extensa y bien capacitada. Todas estas ventajas se intensifican con la participación de los padres y la comunidad. Los profesores generalmente aceptan tomar parte en programas de promoción de la salud como función natural en la escuela y la comunidad, cuando las intervenciones son sencillas y bien planeadas.

Una estrategia de salud escolar integral puede ayudar a los estudiantes, sus familiares, al personal escolar y a los miembros de la comunidad a cuidar de su salud y la de otras personas, y a tomar decisiones y asumir el control de su propia salud. Su ubicación en las escuelas es ideal para favorecer a los desprotegidos, las niñas y los pobres. Si bien el sistema escolar rara vez es universal, su cobertura es muy superior a la de cualquier sistema de salud, especialmente si se desea llegar a los jóvenes, los grupos indígenas y los segmentos pobres de la población.

Para aumentar las probabilidades de éxito en programas de nutrición y salud escolar, cada gobierno debe determinar sus prioridades mediante un análisis de los problemas y necesidades, y un fortalecimiento de los acuerdos intersectoriales que incluya como mínimo a los ministerios de educación y de salud. Siempre que se pueda, deben abarcarse también estrategias de estimulación tem- 
prana, orientadas por conceptos de salud y nutrición para los niños de edad preescolar. En cuanto a los adolescentes, debe enfocarse la enseñanza de habilidades para la vida, especialmente su capacitación para tomar decisiones acerca de su vida sexual y reproductiva y prevenir conductas de riesgo como la violencia y el uso de drogas y tabaco. Otras habilidades importantes son las buenas relaciones interpersonales, el análisis crítico de la publicidad, la comunicación con la familia, la igualdad entre los géneros y la resolución negociada de conflictos.

La OPS y el Banco Mundial trabajarán juntos en esta iniciativa con la meta final de contribuir al desarrollo humano sostenible de las futuras generaciones. Por lo tanto, han acordado fortalecer la capacidad de los gobiernos, las instituciones y las organizaciones para trazar estrategias eficaces, ejecutar programas de salud escolar y de nutrición y vigilar y evaluar su éxito. Se ofrecerán talleres técnicos de alcance regional para crear y probar instrumentos que permitan reconocer y medir los problemas de salud de los escolares y se apoyará a los países para que realicen análisis de su situación y sus necesidades en particular. Una de las actividades más importantes será el apoyo para la elaboración de planes de acción con todos los componentes que requieren en sus diversas etapas. Puesto que muchos países cuentan ya con elementos de salud escolar en marcha, se espera que la iniciativa descrita pueda ejecutarse con bastante facilidad y entusiasmo.

\section{Rondas de las primeras damas}

Las primeras damas hicieron tres rondas de presentaciones en las que informaron de los progresos realizados en sus países en las áreas favorecidas durante cumbres anteriores. A continuación se citan algunos de los comentarios que brindaron. Las primeras damas de Antigua y Barbuda, Brasil, Guyana, Saint Kitts y Nevis, Haití y los Estados Unidos hablaron a favor de la participación y los derechos políticos de la mujer. Recalcó Patricia Bird, de Antigua y Barbuda, que pese a la enorme responsabilidad económica y social que la mujer en general asume, continúa casi sin representación en las decisiones económicas y políticas. En su país, explicó, se reconoce que la integración de la mujer es absolutamente crucial para lograr el desarrollo sostenible en un ambiente democrático estable. La tarea de asegurar la igualdad de género, la participación y el desarrollo de la mujer es un reto continuo para el Directorio de Asuntos Femeninos de su país, que está comprometido a capacitar parlamentarias y consejeras gubernamentales locales.

Hillary Rodham Clinton, de los Estados Unidos, declaró en su discurso que una democracia vi- brante requiere la potenciación y plena participación de las mujeres y que estamos en un momento de la historia lleno de esperanza, propicio para el cambio social. "La democracia", manifestó, "solo puede lograr su potencial más pleno cuando a las mujeres no se les prohíbe por ley, por ignorancia, por tradición o costumbre o por la intimidación, que sus voces sean escuchadas en el momento de votar". También denunció la violencia contra la mujer como un crimen intolerable.

Patricia Escobar de Arzú, primera dama de Guatemala, informó sobre el programa de rehabilitación de niños delincuentes que se está llevando a cabo en su país. Recordó que hubo un tiempo cuando se pensaba que para satisfacer las necesidades de los niños bastaba con proporcionarles abrigo y alimento. Ahora sabemos que no es suficiente. El programa para niños de 8 a 15 años de edad requiere algo más que bienes materiales. Ha sido importante inculcarles valores espirituales y morales que les ayuden a recuperar su autoestima y a sentirse libres, triunfadores, dignos de aprecio, respeto y dignidad. Así es como se construye una cultura de paz, comentó la oradora.

Las primeras damas y otras representantes de Bolivia, Colombia, Cuba, Ecuador, El Salvador, Honduras, México, Paraguay, Perú, República Dominicana, Suriname y Uruguay expresaron interés particular en la salud y la educación y presentaron algunas de las estrategias que se están ejecutando en sus países respectivos. Dora Boyd de Pérez Balladares describió la iniciativa del modelo escolar patrocinada por todas las primeras damas, "Escuelas siglo XXI: formando para la vida", cuyo propósito es fomentar en niños y adolescentes habilidades y conocimientos, actitudes y comportamientos responsables esenciales para la vida. Se refirió al mismo tiempo a la necesidad de crear ambientes saludables que reúnan las condiciones necesarias para garantizar el derecho a un desarrollo humano sostenible de niños y niñas y de adolescentes. Su asesora técnica, Leonor Calderón, presentó el Plan de Acción correspondiente a la iniciativa, el cual fue aprobado.

\section{Escuelas siglo XXI: formando para la vida}

Desde 1993 la OPS trabaja impulsando el enfoque integral de la salud escolar. Como resultado, en muchas escuelas de América Latina y el Caribe hay programas de promoción de la salud y la educación. En 1996 se creó la Red Latinoamericana de Escuelas Promotoras de Salud, que abre una etapa de consolidación regional de la estrategia de trabajo. La iniciativa "Escuelas siglo XXI", presentada por Panamá, pretende fortalecer la capacidad de los países para crear un modelo de escuela que asegure 
la salud y la educación de los escolares de todas las edades, como responsabilidad de todos. Se trata de una inversión que cada sociedad debe hacer para generar, por medio de la capacidad creadora, crítica y productiva de la niñez y la adolescencia, un futuro social y humanamente equilibrado y responsable, con respeto a la diversidad pluricultural y multiétnica del continente.

Las primeras damas abogarán por la visibilidad del tema en la agenda política pública. Dos aspectos importantes serán la creación o el fortalecimiento de comisiones nacionales multisectoriales y la difusión de información para sensibilizar al público con respecto a los objetivos y propuestas de acción de la iniciativa. Las comisiones serán integradas por personal de diversos ministerios (salud, educación, juventud, cultura) y organismos no gubernamentales por medio de talleres, seminarios y otras estrategias de organización. El aspecto informativo se realizará mediante mesas redondas, foros, conferencias de prensa y otras formas de difusión para despertar y mantener el interés en la iniciativa de los miembros del gobierno, la sociedad civil y los formadores de la opinión pública. Para este fin se estimulará también la publicación de una revista que circule en el medio escolar. Cuatro componentes programáticos fundamentales serán 1) el cultivo de ambientes y entornos saludables; 2) la creación de servicios de salud y nutrición integrales; 3) el respeto a los derechos del niño y del adolescente, y 4) la orientación de la educación al desarrollo, la democracia y la paz. Para ello será necesario, entre otros proyectos, renovar los espacios de juego y recreación, tomar medidas de seguridad y de prevención de accidentes, fomentar las relaciones interpersonales sanas y la salud mental de los escolares, establecer quioscos escolares que ofrezcan alimentos sanos y nutritivos, mejorar los sistemas de agua potable y de saneamiento básico, cultivar granjas escolares y enseñar oficios en las escuelas, y promover la actividad deportiva, especialmente para las niñas. La iniciativa entraña una gran riqueza de posibilidades, no solo para los escolares sino también para los maestros, padres y comunidades.

\section{Otros puntos de interés}

Ivy Sylvia Cooke, primera dama de Jamaica, y Marta Larrachea de Frei, de Chile, abogaron por los derechos y la participación de los adultos de mayor edad. La señora de Frei invitó a los presentes a concurrir a la octava cumbre que se celebrará en Chile en 1998.

Antes de clausurarse la reunión, se dio lectura a la Declaración de Panamá, la cual fue suscrita por los presentes como acto final de la reunión.

\section{DECLARACIÓN DE PANAMÁ}

Las primeras damas de las Américas y las representantes designadas por los Gobiernos, reunidas en Panamá del 8 al 9 de octubre de 1997 bajo el lema "Construyamos el futuro de América con derechos humanos y cultura de paz", con el fin de evaluar los logros alcanzados y renovar nuestro compromiso con la solución de los problemas sociales de nuestro Continente, acordamos suscribir la siguiente Declaración:

1. Reconocemos que la problemática social que viven nuestros países nos es común en muchos aspectos y que su solución exige la participación y el compromiso de todos los sectores de la sociedad.

2. Creemos firmemente que la promoción y el respeto de los derechos humanos, el logro de la igualdad y el fortalecimiento de valores como la solidaridad, la tolerancia, la cultura de paz, el pluralismo, la diversidad y el derecho a la autodeterminación de los pueblos son condiciones fundamentales para el desarrollo y la integración en una comunidad de naciones.

3. Comprobamos la existencia de grupos de población sumamente vulnerables que reciben con mayor fuerza el impacto de la pobreza, el desempleo y la desintegración social. Entre estos, los niños, los jóvenes, las mujeres de zonas urbanas y rurales, las personas mayores y las personas con discapacidades requieren de políticas y programas que les faciliten su incorporación al proceso y los beneficios del desarrollo.

4. Reiteramos la voluntad de poner nuestra capacidad como movilizadoras, facilitadoras, gestoras o convocadoras de políticas y programas sociales al servicio de nuestros países, con énfasis en los sectores vulnerables, en plena concordancia con los intereses nacionales de cada país, e inspirándonos en el diálogo, la negociación y el respeto mutuo.

5. Seguiremos teniendo como marco de referencia y de orientación las recomendaciones y decisiones apoyadas por nuestros Gobiernos en la Cumbre Mundial sobre la Infancia en 1990, la Conferencia de las Naciones Unidas sobre el Medio Ambiente y el Desarrollo en 1992, la Declaración de Ginebra sobre la Mujer Rural en 1992, la Conferencia Mundial sobre Derechos Humanos en 1993, la Conferencia Internacional sobre Población y Desarrollo en 1994, la Cumbre de las Américas en 1994, la Cumbre Mundial sobre Desarrollo Social en 1995, la Cuarta Conferencia Mundial de las Naciones Unidas sobre la Mujer en 1995 y la Cumbre Mundial de la Alimentación en 1996. 
6. Conscientes de los acelerados cambios que operan en el mundo de hoy, reiteramos nuestro compromiso de mejorar e incrementar el acceso a los sistemas de educación, capacitación, información y tecnología, a fin de dotar a nuestras poblaciones de las herramientas necesarias para asumir exitosamente la responsabilidad de contribuir a nuestro desarrollo.

7. Reiteramos nuestro compromiso de cumplir nuestros acuerdos sobre:

- Prevención del embarazo precoz;

- Prevención y control del cáncer;

- Eliminación del sarampión;

- Eliminación de todas las formas de violencia;

- Disminución de la morbilidad y mortalidad maternas;

- Cumplimiento de los derechos y deberes de la infancia;

- Mejoramiento de la calidad de los sistemas de educación en la niñez;

- Acceso equitativo de niñas y niños a la educación; y

- Acceso equitativo de la mujer a los procesos de toma de decisiones.

8. Valoramos el trabajo realizado por los países de la Región que apoyan la eliminación del sarampión y de otras enfermedades en las Américas y reiteramos nuestro compromiso de seguir prestando apoyo en ese campo hasta que el sarampión sea erradicado.

9. Continuamos profundamente preocupadas porque persisten la pobreza y la desigualdad entre las mujeres rurales de las Américas. Como un asunto de prioridad urgente, se deben diseñar acciones expeditas para fortalecer la integración de las mujeres rurales en actividades educativas, productivas y comerciales. Estamos seguras de que las instituciones internacionales, privadas y regionales reconocerán su responsabilidad de proveer acceso a la asistencia técnica y financiera para este esfuerzo.

10. Es imprescindible que el proceso de formación en la niñez y la adolescencia incluya el desarrollo de conocimientos, habilidades y sentido de responsabilidad para cuidar íntegramente su salud, para garantizar y promover sus derechos y para fomentar la convivencia, la no discriminación, la igualdad entre hombres y mujeres y la resolución de conflictos a través del diálogo, la comunicación, la negociación y el respeto mutuo.

11. Comprometemos nuestro esfuerzo para contribuir a la erradicación del trabajo infantil, cuyas repercusiones impiden la educación y el desarrollo armónico e integral de los niños y niñas de nuestros países. Por ello solicitamos que se declare un año internacional para la erradicación del trabajo infantil.
12. Reconocemos la urgencia de que todos los países adopten las medidas necesarias para cumplir con la Convención sobre los Derechos del Niño en particular y enfrentar cuanto antes la eliminación del tráfico de niños y niñas para la venta de órganos, la pornografía y la prostitución infantil, que inseparablemente mutilan y laceran su cuerpo y espíritu.

13. Invitamos a los países que aún no lo hayan hecho a ratificar la Convención Interamericana para Prevenir, Sancionar y Erradicar la Violencia contra la Mujer, conocida como la Convención de Belém do Pará, adoptada en 1994.

14. Apoyamos las iniciativas destinadas a la promoción de los derechos humanos para sentar las bases de una verdadera cultura de paz y desarrollo, así como las encaminadas a superar la pobreza en nuestro continente.

15. Reconocemos la valiosa participación y contribución de los organismos internacionales y entidades financieras que han apoyado nuestras iniciativas, haciendo posible la ejecución de proyectos y programas destinados a los sectores más necesitados y vulnerables de nuestras sociedades.

16. Agradecemos y aceptamos el amable ofrecimiento de la primera dama de Chile para recibir la VIII Conferencia de Esposas de Jefes de Estado y de Gobierno de las Américas en 1998.

17. Agradecemos a la Primera Dama de Panamá, al pueblo y al Gobierno de Panamá la amable atención recibida durante nuestra estadía.

Firmada en la Ciudad de Panamá en dos originales, español e inglés, el 9 de octubre de 1997.

\section{SYNOPSIS}

\section{Seventh conference of wives of Presidents of the Americas}

As has been done ever since 1990, when the First Conference of Wives of Presidents and Heads of State was held, first ladies and other women representing more than 30 countries as well as various national and international organizations met in Panama City on 8 and 9 October, 1997. The meeting was a forum for discussing how far the countries have come in the implementation of projects and programs for the benefit of the underprivileged and groups facing discrimination, such as women and children. Attention was brought to bear on the still existing gap between the protective legislation in existence and its enforcement, and there was a renewed commitment to continue to fight for greater equality and justice in the countries of the Americas. The meeting ended with the Panama Declaration, which summarizes the most pressing social goals and the action plan by means of which such goals will be pursued. 